\title{
The ANGRA Neutrino Project
}

\author{
J.C. Anjos ${ }^{1}$, A. F. Barbosa, H. Lima Jr. \\ Centro Brasileiro de Pesquisas Físicas \\ Rua Xavier Sigaud 150, 22290-180, Rio de Janeiro, RJ, Brazil \\ E-mail: janjos@cbpf.br, laudo@cbpf.br, hlima@cbpf.br \\ E. Kemp \\ State University of Campinas \\ Gleb Wataghin Physics Institute, CP 6165, 13083-970, Campinas, SP, Brazil \\ E-mail:kemp@ifi.unicamp.br
}

\section{H. Nunokawa}

Pontifícia Universidade Católica do Rio de Janeiro

R. Marques de São Vicente 225, , Gávea, 22453-900, Rio de Janeiro, RJ, Brazil

E-mail:nunokawa@fis.puc-rio.br

We present the status of the Angra Neutrino project, describing the development of an antineutrino detector aimed to monitor nuclear reactor activity. The experiment will take place at the Brazilian nuclear power plant located in Angra dos Reis. The Angra II reactor, with 4GW of thermal power, will be used as a source of antineutrinos. We expect to observe about one thousand events per day with a detector of one ton scale, placed at about 60 meters of the reactor core. We intend, in a first step, to use the measured neutrino event rate to monitor, with a few per cent accuracy, the thermal power delivered by the reactor. In a second step we intend to determine the fuel isotopic composition through a precise neutrino energy spectrum measurement. In addition to the safeguards issues the project will provide an alternative tool to have an independent control of the reactor delivered thermal power.

10th International Workshop on Neutrino Factories, Super beams and Beta beams Valencia, Spain

30 June - 05 July, 2008

\footnotetext{
1 Speaker. Collaboration members: http://portal.cbpf.br/index.php?page=GruposPesquisa.Pessoal\&grupo=40
} 


\section{Introduction}

In the last ten years there has been a fantastic development in neutrino physics meanwhile important advances has been achieved in the neutrino detection techniques. This has made possible the appearance of potential new technological applications, such as the use of antineutrino detectors for safeguards verification. Indeed, antineutrinos are intensively produced in nuclear reactors and can not be shielded. Since each fissionable isotope has a different antineutrino energy spectrum, the remote detection of the antineutrino flux can in principle reveal the fissile composition of nuclear fuel, giving an indication of the Plutonium content at the end of the fuel cycle. At the same time, as the antineutrino flux is proportional to the reactor thermal power, the flux measurement can provide an independent monitoring of the reactor power at a few per cent level.

\section{The Angra Project: present status}

The Angra Neutirno Project aims to operate a set of neutrino detectors at the Angra dos Reis nuclear complex to measure the neutrino flux emmited by the 4GW PWR nuclear reactor of the Angra-II unit. The antineutrino interactions are from inverse $\beta$-decays. For 1 ton detector we expect rates of $5 \times 10^{2} /$ day $\left(3 \times 10^{4} /\right.$ day $)$ for a distance of $200 \mathrm{~m}(25 \mathrm{~m})$ from the reactor core, enabling to investigate the potential of antineutrino detection for safeguards applications. Further details on the detector systems and geometry can be found elsewhere [1].

We have recently (October 2008) deployed a local laboratory, housed in a container, close to the reactor dome wall (26 $\mathrm{m}$ from the reactor core) to study backgrounds at the surface level. We have also submited to the company that operates the reactor, Eletronuclear, a cooperation agreement and a request to install an underground laboratory. To comply with the site safety requirements, determined by the Eletronuclear, we are proposing two different shaft configurations. The first use only local soil to achieve an overburden about $30 \mathrm{~m}$.w.e. to reduce muon background. The other option is a shallower well with additional layers of heavy material to provide the required overburden.

The final detector design is constrained by the allowed size of the underground laboratory. In Figure 1 we show three possible configurations: a) the standard cilindrical 3 volume design [1], b) a cilindrical 2 volume design with no gamma-catcher and the buffer substituted by thick acrilic walls and c) the "caipirinha" cup 3 volume design with reflective walls and top photomultipliers readout [2]. Simulations are in course to guide the final choice by the best compromise between performance and reduction of the size detector.

The liquid scintillator base is a mixture of dodecane and PXE in the proportion of $4: 1$. The target liquid is loaded with $1 \mathrm{~g} / 1$ of gadolinium to enhance neutron captures. The choice is conservative and based on the well known properties and stability of this mixture that is also used by the Double Chooz collaboration [ref]. An alternative to PXE under consideration is the Linear Alkyl Benzene (LAB) due to its low cost and high flash point (around $140^{\circ} \mathrm{C}$ ). Some samples of LAB added by wavelength shifters were prepared and are under test [3]. 


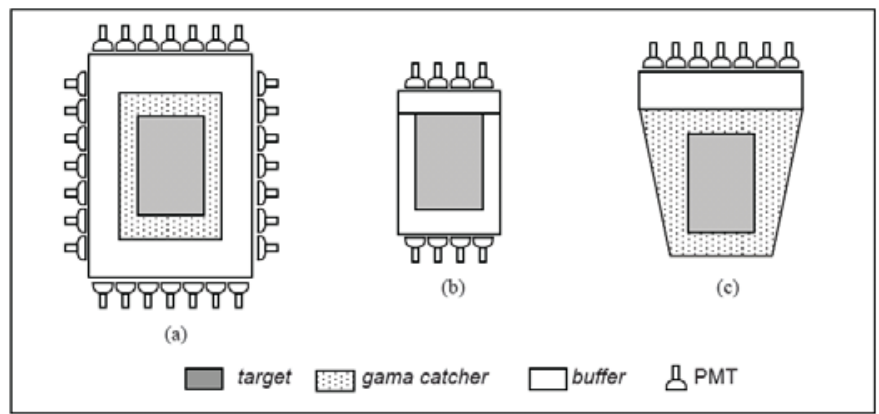

Figure 1: Different detector geometries under study. The choice will be done by the compromise between performance and detector size.

The front-end and DAQ electronics will be made of custom developed modules. The front-end integrates the High Voltage power supply, slow control, PMTs signal preamplification and discrimination. High voltage and slow control parameters will be set and monitored by a microcontroller device accessible by a CAN bus via USB ports. The pre-amplifier circuit [4] provides pulse height gain and shaping to deliver signals to the DAQ 8-channel modules. The DAQ modules are based on VME standards, acting as waveform digitizers and doing time-difference measurements with 100 ps resolution. Similar modules are been developed to be used as the muon electronics of the Double Chooz experiment. Pulse sampling is made by a 10-bit ADC, operating at $250 \mathrm{MHz}$. The DAQ whole information is buffered in FIFO banks. All control functions will be carried out by high-performance FPGAs. Prototypes of the pre-amplifier and 1-channel DAQ circuits are ready with pulse shaping and waveform digitization already implemented.

We are performing simulations of the expected spectra in the detector in different times of the fuel cycle, considering $10 \%$ of sistematic error in energy measurements. The amount of each fuel component is obtained by fitting the neutrino spectrum with the parametrization proposed in [5]. First results shows that at the begining of the cycle we can succesfull extract the ${ }^{235} \mathrm{U}$ fraction $(5 \%$ error) with one month of data taking. After one year of fuel burning, we are observing a sistematic $10 \%$ excess on the obtained fraction. We expect to reduce these errors with further development of data analysis techniques.

\section{References}

[1] J. C. Anjos et al., Angra Neutrino Project: status and plans, Nucl.Phys. B (Proc. Suppl.), 155 (2006) 231; J. C. Anjos et al, Angra dos Reis Reactor Neutrino Oscillation Experiment, Braz. J. of Phys, 36 (2006) 1118.

[2] R. W. McKewon and D. Reyna, A Comparison of the performance of compact neutrino detector designs for nuclear reactor safeguards and monitoring, arXiv: physics/0610257.

[3] .Patrick Pfahler, Basic mixing and purification of liquids scintilators, Angra Note 006-2008, http://www.cbpf.br/ angra/files/angra_notes/AngraNote_006-2008.pdf

[4] A. F. Barbosa, Preliminary simulation study of the front-end electronics for the centraldetector PMTs, Angra Note 001-2008, http://www.cbpf.br/ hlima/files/angra/AngraNote 001-2007.pdf

[5] P.Huber and T. Schwetz, Precison spectroscopy with reactor anti-neutrinos, Phys.Rev. D70 (2004) 073014 [hep-ph/0403068]. 